\title{
Serum anti-myomegalin antibodies in patients with esophageal squamous cell carcinoma
}

\author{
HIDEAKI SHIMADA ${ }^{1}$, MARI KUBOSHIMA ${ }^{1,2}$, TOORU SHIRATORI ${ }^{1}$, \\ YOSHIHIRO NABEYA ${ }^{1}$, ATSUSHI TAKEUCHI ${ }^{3}$, HIROAKI TAKAGI ${ }^{4}$, FUMIO NOMURA ${ }^{3}$, \\ MASAKI TAKIGUCHI ${ }^{2}$, TAKENORI OCHIAI ${ }^{1}$ and TAKAKI HIWASA ${ }^{2}$ \\ Departments of ${ }^{1}$ Frontier Surgery, ${ }^{2}$ Biochemistry and Genetics, and ${ }^{3}$ Molecular Diagnosis, Chiba University, \\ Graduate School of Medicine, Chiba 260-8670; ${ }^{4}$ ProteinExpress, Choshi Laboratory, Choshi, Chiba, Japan
}

Received August 11, 2006; Accepted October 16, 2006

\begin{abstract}
In order to identify new serum markers of esophageal squamous cell carcinoma (SCC), we performed serological identification of antigens by recombinant cDNA expression cloning (SEREX). E. coli was transformed with a $\lambda$ ZAPII phage cDNA library prepared from mRNA of an esophageal cancer cell line (T.Tn), and IPTG-induced cDNA products were screened for interaction with antibodies in allogeneic sera of patients with esophageal SCC. We identified myomegalin (MMGL, phosphodiesterase 4D interacting protein/PDE4DIP) as a new SEREX antigen for esophageal SCC. Western blot analysis revealed that serum anti-myomegalin antibodies (s-MMGL-Abs) were present in $43(47 \%)$ of 91 patients, but in only one $(2.2 \%)$ of 45 healthy controls. Of the 21 patients with stage I disease, 8 (38\%) were sero-positive. The positive rate of s-MMGL-Abs was greater than those of other conventional tumor markers. Reverse transcription-PCR analysis suggested that alternative splicing from myomegalin variant 1 to variant 5 may explain, in part, the development of s-MMGL-Abs. Although the presence of s-MMGL-Abs was not related to any clinicopathological features of the patients, multivariate analysis indicated that the presence of s-MMGL-Abs was significantly associated with a favorable prognosis. Consequently, s-MMGL-Abs may be a useful tumor marker to diagnose and establish a prognosis in patients with esophageal SCC.
\end{abstract}

Correspondence to: Dr Takaki Hiwasa, Department of Biochemistry and Genetics, Chiba University, Graduate School of Medicine, Inohana 1-8-1, Chuo-ku, Chiba 260-8670, Japan

E-mail: hiwasa_takaki@faculty.chiba-u.jp

Abbreviations: IPTG, isopropyl B-D-thiogalactoside; PBS, phosphate-buffered saline; RT-PCR, reverse transcriptase-PCR; SCC, squamous cell carcinoma; SEREX, serological identification of antigens by recombinant cDNA expression cloning; s-MMGL-Abs, serum anti-myomegalin antibodies

Key words: SEREX, myomegalin, esophageal squamous cell carcinoma, tumor marker

\section{Introduction}

Esophageal squamous cell carcinoma (SCC) is a deadly disease. Late presentation in a group of elderly patients with co-morbid illnesses resulted in a poor outcome $(1,2)$. The aggressive behavior of esophageal SCC has been associated with systemic involvement at diagnosis, and the poor prognosis has been attributed largely to a delay in diagnosis (2). Although several serum markers have been reported to be clinically useful for diagnosing esophageal SCC $(3,4)$, these serum markers are limited in terms of detecting early esophageal cancers, except the serum p53 $\operatorname{IgG}$ antibody $(5,6)$. In order to identify new tumor-associated antigens that may generate new serum markers for esophageal SCC, we used the method of serological identification of antigens by recombinant cDNA expression cloning (SEREX) which involves the immunoscreening of cDNA libraries prepared from tumor specimens using autologous or allogeneic sera (7). Chen et al (8) and our group (9-13) have succeeded in the identification of several tumor antigens for esophageal SCC. The identification of human tumor antigens recognized by the autologous host is yielding an array of target molecules for the diagnosis, monitoring, and immunotherapy of human cancer (14-16).

In the present study, we identified myomegalin as a new SEREX antigen of esophageal SCC, observed that serum anti-myomegalin antibodies (s-MMGL-Abs) were present frequently in esophageal SCC patients, and associated s-MMGL-Abs with a favorable prognosis in patients with esophageal SCC.

\section{Materials and methods}

Human esophageal squamous cell carcinoma cells and tissues. Seven human esophageal squamous cell carcinoma cell lines and one human immortalized cell line were used. T.Tn was obtained from the Japan Cell Research Bank (Ibaraki, Japan) (17). TE-10 was obtained from Cancer Cell Repository, Institute of Development, Aging and Cancer, Tohoku University, Sendai, Japan (18). The YES-1, YES-2, YES-4, YES-5 and YES-6 were kindly provided by Drs M. Oka and T. Abe (Yamaguchi University Graduate School 
of Medicine) (19). WI-38 human fibroblasts were obtained from Riken Bioresource Center (Ibaraki, Japan). Cells were cultured in Dulbecco's modified Eagle's medium (SigmaAldrich, MO, USA), supplemented with $10 \%$ heat-inactivated fetal bovine serum. EN-60 cells are human esophageal keratinocytes immortalized via E6 and E7 of human papillomavirus type 16 (20). Normal esophageal tissue was obtained from patients with esophageal SCC who underwent subtotal esophagectomy. This work was approved by the Ethics Committee of Chiba University Graduate School of Medicine. The collection of tissues and sera was agreed upon by the patients and healthy donors, each provided a written, informed consent.

Sera from patients with esophageal SCC and healthy donors. Sera were obtained from 45 healthy volunteers and 91 patients with esophageal SCC who represented various stages of disease progression before treatment. The patients consisted of 84 men (92\%) and 7 women $(8 \%)$, with a median age of 67 years (range 39-86 years). A total of 48 patients received surgery and 43 patients received chemoradiation therapy. Surgically treated patients were pathologically classified according to the TNM staging system defined by Union of International Cancer classifications (UICC) (21) using resected specimens. Non-surgically treated patients were clinically classified according to TNM/UICC staging system using computed examination, positron emission tomography, endoscopic ultrasonography and cervical ultrasonography examinations (22). The patient cohort included 21 with stage I, 22 with stage II, 35 with stage III, and 13 with stage IV cancer. Each serum sample was centrifuged at $3,000 \times \mathrm{g}$ for $5 \mathrm{~min}$ and the supernatant was stored at $-80^{\circ} \mathrm{C}$ until use. Repeated thawing and freezing of samples were avoided. After treatment, all patients underwent clinical examination and imaging studies on a regular basis for at least 60 months or until death. All patients were followed up for at least 2 years, 51 of 91 patients had died by the end of 2005 .

Enzyme immunoassay for CEA, CYFRA21-1, SCC-Ag and serum p53 antibodies. Serum CEA and CYFRA-Ag concentrations were accessed using the Enzymun-Test CEA and Enzymun-Test CYFRA21-1 (Boehringer Mannheim, Mannheim, Germany), respectively. SCC-Ag levels were quantitated in serum using the SCC Test (Abbott Laboratories, Abbott Park, IL). The cut-off values for serum CEA, CYFRA-Ag, and SCC-Ag levels were $4.6 \mathrm{ng} / \mathrm{ml}, 2.57 \mathrm{ng} / \mathrm{ml}$ and $1.5 \mathrm{ng} / \mathrm{ml}$, respectively, in accordance with the manufacturer's instructions. The specificity at these cut-off values is $95 \%(3,4)$.

Serum p53-Ab was assessed by enzyme-linked immunosorbent assay using the anti-p53 ELISA Kit II (Medical \& Biological Laboratories, Nagoya, Japan; Trade name: MESACUP anti-p53 Test) as described previously $(5,6)$. All samples were considered positive at $1.3 \mathrm{U} / \mathrm{ml}$. The specificity at these cut-off values is $95 \%$ (6).

SEREX screening. Total RNA was prepared from T.Tn cells by the acid guanidinium thiocyanate-phenolchloroform method and purified poly $(\mathrm{A})^{+}$RNA was obtained using the Oligotex- $\mathrm{dT}_{30}$ (Super) mRNA Purification Kit (Takara
Biochemicals, Kyoto, Japan) according to the manufacturer's instructions (23). Double-stranded cDNA was synthesized by conventional procedures and ligated into the EcoRI-XhoI site of $\lambda$ ZAP II phage. The original library size consisted of $1.8 \times 10^{6}$ independent clones. The immunological screening method used was a modification from that used in a previously published study $(7,8)$. E. coli XL1-Blue MRF' was infected with $\lambda$ ZAP II phage and the expression of resident cDNA clones was induced following blotting of infected bacteria onto NitroBind nitrocellulose membranes (Osmonics Inc., Minnetonka, MN) that had been pre-treated with $10 \mathrm{mM}$ isopropyl B-D-thiogalactoside (IPTG) (Wako Pure Chemicals, Osaka, Japan) for $30 \mathrm{~min}$. The membranes with adhered bacterial proteins were washed three times with TBS-T [20 mM Tris- $\mathrm{HCl}$ ( $\mathrm{pH} 7.5$ ), $0.15 \mathrm{M} \mathrm{NaCl}$ and $0.05 \%$ Tween-20], and blocking was performed by treatment with $1 \%$ protease-free bovine serum albumin (Wako Pure Chemicals) in TBS-T for $1 \mathrm{~h}$. The membranes were exposed to 1:2000-diluted serum for $1 \mathrm{~h}$. After washing with TBS-T three times, the membranes were treated for $1 \mathrm{~h}$ with 1:5000-diluted alkaline phosphatase-conjugated goat anti-human IgG (Jackson ImmunoResearch Laboratories, West Grove, PA). Positive reactions were detected by incubation in a color development solution [100 mM Tris- $\mathrm{HCl}$ (pH 9.5), $100 \mathrm{mM} \mathrm{NaCl}$ and $5 \mathrm{mM}$ $\mathrm{MgCl}_{2}$ ] containing $0.3 \mathrm{mg} / \mathrm{ml}$ of nitroblue tetrazolium chloride (Wako Pure Chemicals) and $0.15 \mathrm{mg} / \mathrm{ml}$ of 5-bromo-4chloro-3-indolylphosphate (Wako Pure Chemicals). Positive clones were re-cloned twice to obtain monoclonality.

Sequence analysis of identified antigens. Monoclonalized phage cDNA clones were converted to pBluescript phagemids by in vivo excision using the ExAssist helper phage (Stratagene, La Jolla, CA). Plasmid DNA was obtained from the E. coli SOLR strain following transformation by the phagemid. The cDNA inserts were sequenced by the dideoxy chain termination method using the DNA Sequencing BigDye ${ }^{\mathrm{TM}}$ Terminator kit (Applied Biosystems, Foster City, CA) and the ABI PRISM 3700 DNA Analyzer (Applied Biosystems). Sequences were searched for homology with identified genes or proteins contained within public sequence databases using the NCBI-BLAST algorithm.

Western blot analysis. E. coli JM109 cells that contained cDNA clones recombined in pBluescript II were cultured with or without $0.1 \mathrm{mM}$ IPTG for $2.5 \mathrm{~h}$. Cells were then washed with phosphate-buffered saline (PBS) and lysed in SDS sample buffer followed by incubation at $100^{\circ} \mathrm{C}$ for $3 \mathrm{~min}$. The lysates were analyzed by Western blotting using sera from 91 patients with esophageal SCC, 16 patients with gastric cancer, 16 patients with colorectal cancer and 45 healthy donors.

Reverse transcriptase-PCR (RT-PCR). Total cellular RNA was isolated from cultured cells and normal and carcinoma tissues of the esophagus using the AquaPure RNA Isolation kit (Bio-Rad, Hercules, CA). Total RNA of normal esophageal keratinocytes was also purchased from Cybrdi (Gaithersburg, $\mathrm{MD})$. Reverse transcription was performed with an oligo(dT) ${ }_{20}$ primer using the ThermoScript RT-PCR System (Invitrogen, Carlsbad, CA). The presence of myomegalin transcripts was 
examined via PCR amplification using the following primers: MMGLv1-742F, 5'-TGGGAAGATGTACCAGGAGAC-3'; MMGLv1-1023R, 5'-GAGTTCTGCCAAGTGGGAATC-3'; MMGLv1-3029F, 5'-TACGCAGTCGGCTAGAAGAAG-3'; MMGLv1-3368R, 5'-TCCTCCTCAGCTTTCCTCTTC-3'; MMGLv4-1032R, 5'-CTCTTGCTTGCCGCTATTTGC-3'; and MMGLv5-1697F, 5'-CACCTGTGAGTTATCCCTT GG-3'. Actin-specific PCR primers (sense, 5'-GGATCAG CAAGCAGGAGTATG-3'; antisense, 5'-GAGAAGTGGG GTGGCTTTTAG-3') were used as positive controls. PCR was performed using KOD-Plus-DNA polymerase (Toyobo, Osaka, Japan) as follows: an initial denaturation step at $94^{\circ} \mathrm{C}$ for $3 \mathrm{~min}$, followed by 30 cycles of denaturation at $94^{\circ} \mathrm{C}$ for $30 \mathrm{sec}$, annealing at $59^{\circ} \mathrm{C}$ for $30 \mathrm{sec}$, and extension at $68^{\circ} \mathrm{C}$ for $30 \mathrm{sec}$, with a final extension of $5 \mathrm{~min}$ at $68^{\circ} \mathrm{C}$.

Immunohistochemical analysis. Polyclonal anti-myomegalin serum was developed by immunizing rabbits with glutathione-S-transferase-myomegalin fusion protein that contained a region of the human KIAA0477/myomegalin protein (between amino acid positions 949 and 1148). The anti-sera were obtained 102 days after the first immunization and IgG was purified by using Protein A-Agarose.

Immunohistochemical staining was performed using the Histofine SAB-PO Kit (Nichirei Corporation, Tokyo, Japan) as described (24). In brief, $4-\mu$ m-thick sections were deparaffinized in xylene, dehydrated through graded alcohol concentrations and incubated in citrate buffer $(\mathrm{pH}$ 6.0) for $5 \mathrm{~min}$ in a household microwave oven at $800 \mathrm{~W}$. After microwave exposure, the slides were allowed to cool to room temperature. The slides were briefly washed with PBS and incubated for 15 min with $3 \%$ hydrogen peroxide in methanol to block endogenous peroxidase activity. The antibodies to myomegalin were diluted 1:250 and incubated for $24 \mathrm{~h}$ at $4^{\circ} \mathrm{C}$. Biotinylated anti-rabbit IgG antibody (Dako Cytomation, Carpinteria, CA) at a dilution of 1:500 was used as the second antibody. After incubation in avidin-biotin complex solution (Dako Cytomation), the staining was developed by the diaminobenzidine method, followed by counterstaining with Mayer's haematoxylin. The stained sections were evaluated at a high magnification $(\times 400)$. Staining was considered positive for myomegalin when more than $10 \%$ of the cells were stained strongly.

Statistical analyses. Fisher's exact probability test was used to determine the significance of differences between the two groups. Survival probabilities were calculated by the KaplanMeier method. Differences between groups were determined using the log-rank test. The influence of each significant predictor identified by univariate analysis was assessed by multivariate analysis using Cox's proportional hazards model. All statistical analyses were carried out using the StatView program version 5.0 for Windows (SAS Institute Inc., Cary, NC). The difference was considered statistically significant when the $\mathrm{P}$-values were $>0.05$.

\section{Results}

Isolation of myomegalin cDNA by SEREX. The phage expression library was constructed using mRNA derived

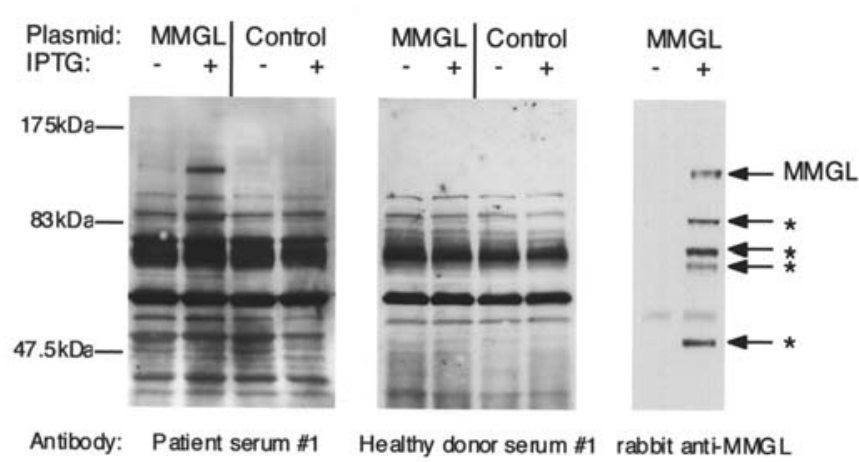

Figure 1. The presence of s-MMGL-Abs in patients with esophageal SCC. $E$. coli containing myomegalin cDNA plasmid (MMGL) or empty pBluescript II vectors (Control) were treated with (+) or without (-) IPTG for $2.5 \mathrm{~h}$, and the total cell lysate was subjected to Western blot analysis using sera of esophageal cancer patient \#1 and healthy donor \#1 or rabbit anti-myomegalin antibody (anti-MMGL). Arrows indicate the IPTG-dependent specific bands, which represent myomegalin cDNA products or their degradation intermediates. $120 \mathrm{kDa}$ is the size of myomegalin estimated from cDNA sequence, and the smaller sizes of approximately 85, 75, 70 and $50 \mathrm{kDa}$ indicated by asterisks may represent degradation products.

from the human esophageal squamous cell line, T.Tn. A total of $5 \times 10^{6} \mathrm{cDNA}$ clones were screened using sera from five esophageal cancer patients, and $>100$ reactive clones were isolated. DNA sequence analysis and a search for homologous sequence in NCBI-accessible databases revealed that this grouping consisted of 40 independent clones. One of the isolated cDNA clones was transcript variant 5 of myomegalin (phosphodiesterase 4D interacting protein/PDE4DIP/ CMYA2/ KIAA0477; GenBank accession number: NM_001002811; UniGene: Hs.528503), which was originally cloned by Verde et $a l$ as a novel protein that interacts with the cyclic nucleotide phosphodiesterase, PDE4D (25).

Presence of $s-M M G L-A b s$ in patients with esophageal SCC. To examine for the presence of s-MMGL-Abs, Western blotting was performed using allogeneic sera obtained from 91 patients with esophageal SCC. An example is presented in Fig. 1. IPTG-pre-treatment of the bacteria induced specific cDNA expression. A positive reaction at $120 \mathrm{kDa}$ was observed only when the extract of IPTG-treated myomegalincDNA-containing bacteria was probed with serum IgG of patient $\# 1$, but not with that of healthy donor \#1. E. coli that contained the control empty plasmid showed no apparent specific reaction after IPTG treatment. The IPTG-dependent $120-\mathrm{kDa}$ protein appeared to be myomegalin because it also reacted with rabbit polyclonal anti-myomegalin antibody, and its size was similar to the product of cloned myomegalin cDNA (1,102 amino acids). Thus, the results shown in Fig. 1 indicate that patient \#1 was positive for s-MMGL-Abs, while healthy donor \#1 was negative.

Clinicopathological features of $s-M M G L-A b s$ in the patients with esophageal SCC. Based on the criteria shown above, sera from 43 (47\%) out of 91 patients with esophageal SCC were positive for s-MMGL-Abs. On the other hand, serum from only one $(2.2 \%)$ of the 45 healthy donors contained a detectable amount of s-MMGL-Abs. Although female patients 
Table I. Relationship between s-MMGL-Abs and the clinicopathological variables in 91 patients with squamous cell carcinoma.

\begin{tabular}{lccc}
\hline Variables & $\begin{array}{c}\text { No. of } \\
\text { patients }\end{array}$ & $\begin{array}{c}\text { No. of patients } \\
\text { with s-MMGL-Abs } \\
\text { (positive rate \%) }\end{array}$ & P-value $^{\mathrm{b}}$ \\
& &
\end{tabular}

\section{Gender}

Male

Female

84

$37(44)$

$6(86)$

Age

$<65$

$\geq 65$

$19(45)$

24 (49)

Location

$\begin{array}{lll}\text { Upper } & 18 & 11(61) \\ \text { Lower } & 73 & 32(44)\end{array}$

Tumor size

$\begin{array}{lll}<50 \mathrm{~mm} & 42 & 22(53) \\ \geq 50 \mathrm{~mm} & 49 & 21(43)\end{array}$

Tumor depth

$\begin{array}{lrrr}\text { T1 } & 28 & 11(39) & 0.430 \\ \text { T2-T4 } & 63 & 32(51) & \\ \text { N factor } & & & \\ \text { N0 } & 28 & 30(48) & 0.999 \\ \text { N1 } & 63 & & \\ \text { M factor } & & 76(46) & 0.828 \\ \text { M0 } & 78 & & \\ \text { M1 } & 13 & & \\ \text { UICC stage } & & 35(54) & 0.477 \\ \text { I } & 21 & & \\ \text { II, III, IV } & 70 & \end{array}$

Treatment

modality

\begin{tabular}{llll} 
Surgery & 48 & $27(56)$ & 0.108 \\
CRT $^{\mathrm{a}}$ & 43 & $16(37)$ & \\
\hline
\end{tabular}

${ }^{\mathrm{a}} \mathrm{CRT}$, chemoradiation therapy; ${ }^{\mathrm{b}} \mathrm{P}$-values were calculated by Fisher's exact probability test.

showed a relatively high incidence of s-MMGL-Abs, there was no statistically significant association between the presence of s-MMGL-Abs and the clinicopathological features of the patients (Table I). The positive rates of s-MMGL-Abs, according to TNM stages, were stage I in $38 \%$, stage II in $45 \%$, stage III in $51 \%$, and stage IV in $54 \%$ (Fig. 2A). The positive rate of s-MMGL-Abs $(47 \%)$ was higher than those of other conventional tumor markers, such as SCC-Ag (38\%), s-p53-Abs (38\%), CYFRA21-1 (24\%), and CEA (23\%) ( $\mathrm{P}=0.29$ for $\mathrm{SCC}-\mathrm{AG}$ and s-p53-Abs, $\mathrm{P}<0.01$ for CEA and CYFRA21-1; Fig. 2B). The sensitivity was improved by using
(A)

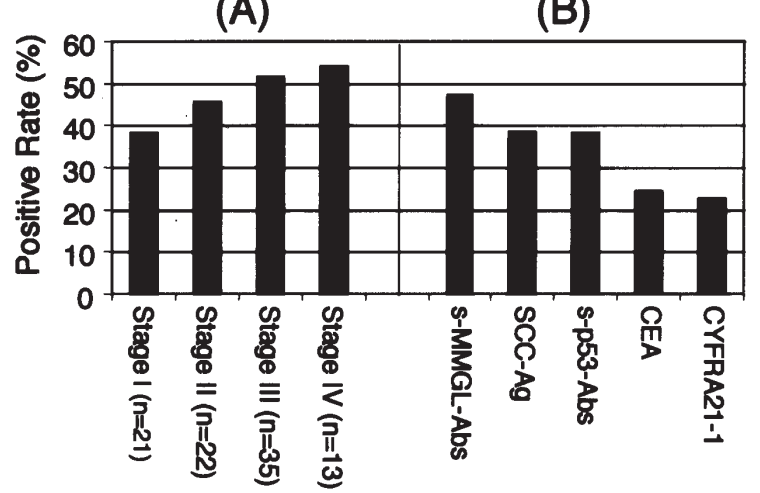

Figure 2. Comparison of positive rates of s-MMGL-Abs. Shown are the positive rates of s-MMGL-Abs in the patients with esophageal SCC according to TNM/UICC stage (A), and the positive rates of s-MMGL-Abs and conventional tumor markers $(\mathrm{B})$.

conventional markers combined with s-MMGL-Abs to $69 \%$ with s-p53-Abs, 69\% with SCC-Ag, 76\% with CYFRA21-1, and $78 \%$ with CEA (data not shown).

The s-MMGL-Abs were tested further using serum samples from patients with gastric or colon cancer. s-MMGL-Abs were positive in $12.5 \%(2 / 16)$ of patients with gastric cancer and in $37.5 \%(6 / 16)$ of patients with colon cancer. Thus, the development of s-MMGL-Abs was not limited in esophageal SCC, but was observed in other cancers, showing the possibility for application of s-MMGL-Abs as a marker of other cancers.

Prognostic significance of $s-M M G L-A b s$ in patients with esophageal SCC. Kaplan-Meier plotting showed that seropositive patients showed better survival rates than seronegative patients, although the difference was not significant ( $\mathrm{P}=0.165$, Fig. 3). All clinicopathological factors listed in Table I were tested by multivariate analysis and independent prognostic factors were identified using Cox's proportional hazards model. After adjusting for tumor stages, the presence of s-MMGL-Abs was shown to be an independent predictor for favorable survival in patients with esophageal SCC (Table II).

Expression of myomegalin in esophageal SCC. The expression levels of myomegalin mRNA in esophageal cell lines were examined by RT-PCR analysis. A total of 27 alternative splicing variants are known for myomegalin/ PDE4DIP mRNA, of which 9 can be translated into proteins (http://www.ensembl.org/Homo_sapiens/index.html). Our preliminary experiments suggested that the myomegalin variants 1, 3, 4 and 5 were expressed more or less in esophageal cells, and, therefore, these variants were examined in detail. The results revealed that the expression of myomegalin variant 1 was observed clearly in normal keratinocytes, immortalized esophageal keratinocytes and T.Tn cells, but was diminished considerably in other esophageal carcinoma cell lines (Fig. 4). Variant 1 was also expressed in WI-38 normal fibroblasts, but not in MKN74 gastric cancer, DLD1 colon carcinoma, HepG2 hepatoma and PC6 lung 


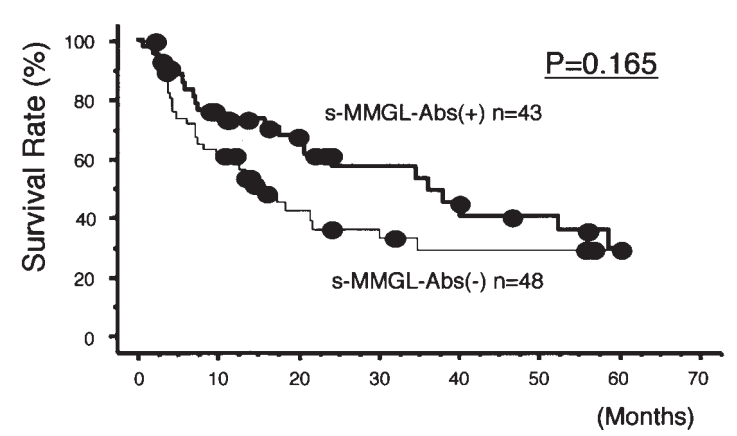

Figure 3. Survival curves and presence of s-MMGL-Abs in 91 patients with esophageal SCC. The bold line indicates the survival of the patients with s-MMGL-Abs $(n=43)$. The thin line indicates the survival of the patients without s-MMGL-Abs $(n=48)$. Circles depict survivors. Statistical significance was determined by the log-rank test.

Table II. Prognostic analysis of patients with esophageal squamous cell carcinoma.

\begin{tabular}{lcccc}
\hline Variables & $\begin{array}{c}\text { Uni- } \\
\text { variate } \\
\text { P-value }\end{array}$ & $\begin{array}{c}\text { Multi- } \\
\text { variate } \\
\text { P-value }\end{array}$ & $\begin{array}{c}\text { Hazard } \\
\text { ratio }\end{array}$ & \\
& & & \\
\hline
\end{tabular}

\section{Gender}

Male

Female

0.773

0.025

1.26-15.15

Age

$<65$

$\geq 65$

0.236

0.148

0.86-2.77

Location

Upper

\section{Lower}

0.159

0.354

1.56

$0.61-3.97$

Tumor size

$<50 \mathrm{~mm}$

$\geq 50 \mathrm{~mm}$

$<0.001$

0.143

1.68

0.84-3.34

Tumor depth

T1

T2-T4

$<0.001$

0.039

$2.867 \quad 1.06-7.81$

$\mathrm{N}$ factor

No

N1

$$
0.001
$$

0.039

2.56

$1.05-5.65$

$\mathrm{M}$ factor

M0

M1

0.099

0.198

1.70

0.76-3.79

s-MMGL-Abs

(+)

(-)

0.165

0.046

1.87

1.01-3.44

carcinoma cells (Fig. 4, data not shown). Thus, the expression of myomegalin variant 1 mRNA was low in most tumor

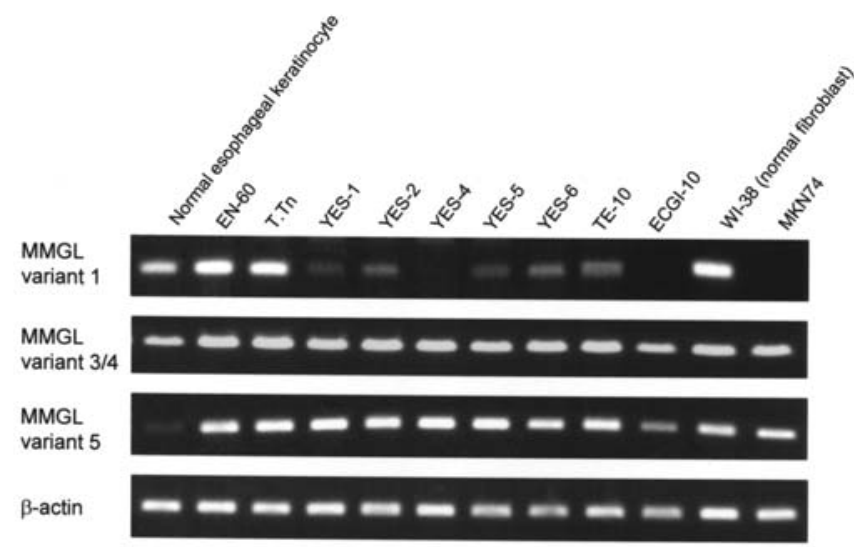

Figure 4. The expression of myomegalin mRNA in normal esophageal keratinocytes and cultured cells. The expression of myomegalin (upper three panels) and $\beta$-actin (lowest panel) was examined by RT-PCR. EN-60 cells are immortalized esophageal cells. Esophageal carcinoma cell lines used were T.Tn, YES-1, YES-2, YES-4, YES-5, YES-6, TE-10 and ECGI-10. WI-38 cells are normal fibroblasts and MKN-74 is a gastric cancer cell line. Primers used were MMGLv1-3029F and MMGLv1-3368R for variant 1; MMGLv1-742F and MMGLv4-1032R for variants 3/4; MMGLv5-1697F and MMGLv1-1023R for MMGL variant 5. For control, 3 -actin primers were used.

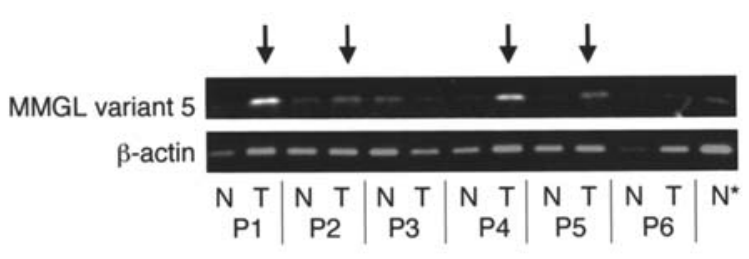

*normal esophageal keratinocyte

Figure 5. Expression of myomegalin mRNA in normal and esophageal SCC tissues. The expression of myomegalin (upper panel) and B-actin (lower panel) was examined by RT-PCR for specimens of normal $(\mathrm{N})$ and carcinoma (T) tissues resected from patients 1 to 6 (P1-P6). Primers used were MMGLv5-1697F and MMGLv1-1023R for MMGL variant 5. "N represents the products from RNA of normal esophageal keratinocytes obtained from Cybrdi. Arrows indicate the higher expression levels of myomegalin mRNA in carcinoma tissues compared to that in the normal tissues of the same patients.

cell lines, except T.Tn cells. On the other hand, myomegalin variants 3 and 4 were expressed ubiquitously in all of the cells examined. The expression of myomegalin variant 5 was observed in most of the cell lines examined, but was noticeably low in normal keratinocytes. The expression levels of myomegalin mRNA in normal esophageal tissues and esophageal carcinoma were also examined (Fig. 5). A higher expression level of myomegalin mRNA was observed in carcinoma tissues compared to that in normal tissues in 4 of 6 patients with esophageal SCC.

Next, we investigated the expression and distribution of myomegalin protein by immunohistochemical analysis in the resected tumor specimens from s-MMGL-Abs-positive patients. The nuclei of each tumor cell showed high immunoreactivity to anti-myomegalin antibodies, whereas normal 


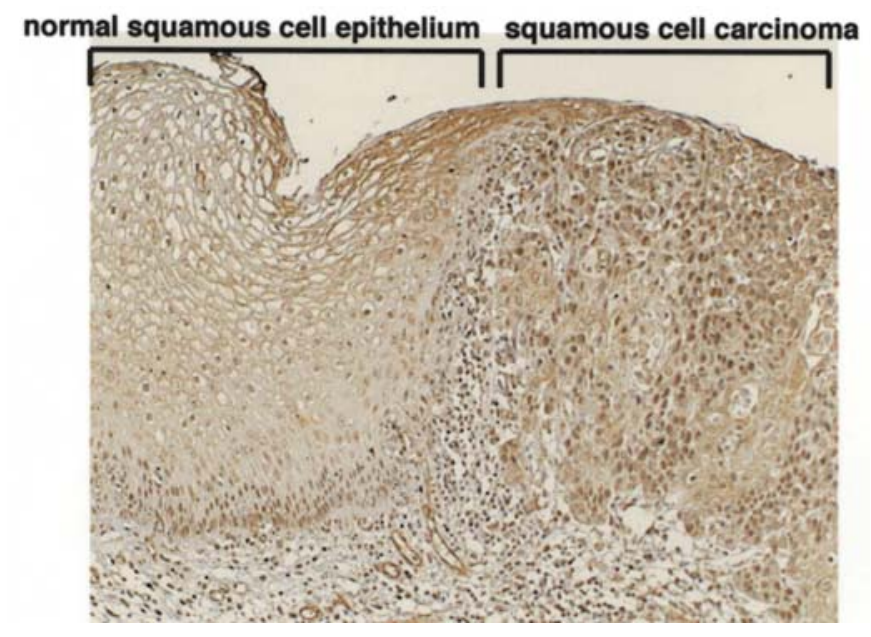

Figure 6. Immunoperoxidase staining of formalin-fixed paraffin-embedded esophageal carcinoma tissues using anti-myomegalin antibody. Original magnification, $\times 400$.

esophageal tissue showed only weak immunoreactivity, with minimal expression being evident only at the basal lamina (Fig. 6).

\section{Discussion}

SEREX screening has been performed in esophageal carcinoma by Chen et al, who identified NY-ESO-1 (8). SEREX antigens are thought to be good candidates for the development of tumor specific vaccination therapies. We have previously reported 21 SEREX antigens of esophageal SCC (9-13). In the present study, we identified myomegalin as a new SEREX antigen of great interest.

Myomegalin is composed of $\alpha$-helical and coiled-coil structures, with domains shared with microtubule-associated proteins and a leucine zipper (LZ) identical to that found in the Drosophila centrosomin (25). Myomegalin has also been designated as PDE4DIP because of its binding to the cyclic nucleotide phosphodiesterase, PDE4D (26). The localization of myomegalin in the Golgi/centrosome suggests that this protein plays a part in transducing cAMP signals in these organelles. Of relevance for this report, myomegalin encodes several putative oligomerization domains capable of activating platelet-derived growth factor receptor $B$ (PDGFRB). A patient with myeloproliferative disorder was shown to have PDGFRB fused to the PDE4DIP isoform lacking the LZ domain (KIAA0477) (26). Therefore, the aberrant expression of myomegalin variants may be involved in carcinogenesis.

Myomegalin transcripts consist of 27 variants with up to 46 exons, of which 9 variants can be translated into proteins (http://www.ensembl.org/Homo_sapiens/index.html). The results of RT-PCR revealed that the expression levels of myomegalin variant 5 were higher in esophageal SCC cell lines and tissues than in non-cancerous cells and tissues (Figs. 4 and 5). On the other hand, the levels of myomegalin variant 1 was diminished in most tumor cell lines compared to that in non-cancerous cells (Fig. 4). Thus, alternative splicing of the myomegalin gene may account for the development of autoantibodies against myomegalin variant 5 .
We evaluated the clinicopathological significance of the presence of s-MMGL-Abs in patients with esophageal SCC. s-MMGL-Abs were present in $47 \%$ of patients with esophageal SCC. This positive rate of s-MMGL-Abs was higher than that observed using other tumor markers such as SCC-Ag, s-p53-Abs, CEA and CYFRA21-1. Moreover, s-MMGL-Abs were present in $38 \%$ of patients with stage I disease (Fig. 2). Therefore, s-MMGL-Abs may be a useful marker to detect early esophageal SCC. Apparent absence of association of s-MMGL-Abs with other serum markers led to combinatory assays that increased the positive rates.

Although the patients with s-MMGL-Abs showed advanced disease, the long-term survival of sero-positive patients was better than that of sero-negative patients. Multivariate analysis showed that the presence of s-MMGL-Abs contributed significantly as a favorable prognostic predictor for long-term survival (Table II). These findings suggest that s-MMGL-Abs may have a role in the suppression of tumor growth. Based on these findings, myomegalin may be proposed as a new target for immunotherapy. Interestingly, tissue distribution of the myomegalin variants varies greatly (27), and a short 2.4-kb mRNA of myomegalin is specifically expressed in testis (25). The next step in examining SEREX-defined antigens should be the analysis of the $\mathrm{CD}^{+}$and $\mathrm{CD}^{+} \mathrm{T}$-cell response directed against these antigens $(28,29)$. While limited immunotherapy has been reported for esophageal SCC, the clinical application of these tumor antigens, such as in cytotoxic $\mathrm{T}$ lymphocyte therapy, is promising $(30,31)$. Our results suggest that myomegalin may be a prime candidate for such studies.

\section{Acknowledgements}

We are grateful to Drs Masaaki Oka and Toshihiro Abe (Yamaguchi University Graduate School of Medicine) for providing the YES esophageal carcinoma cell lines. We also thank Ms. Ayumi Shioya, Masae Suzuki and Saori Yoshida for excellent technical assistance. This work was partly supported by the Grant for 21st Century COE (Center of Excellence) Program and a Grant-in-Aid for Scientific Research from the Ministry of Education, Culture, Sports, Science and Technology of Japan.

\section{References}

1. The Japanese Society for Esophageal Diseases: Comprehensive registry of esophageal cancer in Japan, 1999. Esophagus 2: 43-69, 2005

2. Daly JM, Karnell LH and Menck HR: National cancer data base report on esophageal carcinoma. Cancer 78: 1820-1828, 1996.

3. Shimada H, Nabeya Y, Okazumi S, Matsubara H, Miyazawa Y, Shiratori T, Hayashi H, Gunji Y and Ochiai T: Prognostic significance of serum CYFRA21-1 in the patients with esophageal squamous cell carcinoma. J Am Coll Surg 196: 573578,2003

4. Shimada H, Nabeya Y, Okazumi S, Matsubara H, Shiratori T, Gunji Y, Kobayashi S, Hayashi H and Ochiai T: Prediction of survival with squamous cell carcinoma antigen in patients with resectable esophageal squamous cell carcinoma. Surgery 133: 486-494, 2003.

5. Shimada H, Takeda A, Arima M, Okazumi S, Matsubara H, Nabeya Y, Funami Y, Hayashi H, Gunji Y, Suzuki T, Kobayashi S and Ochiai T: Serum p53 antibody is a useful tumor marker in superficial esophageal squamous cell carcinoma. Cancer 89: 1677-1683, 2000. 
6. Shimada H, Ochiai $\mathrm{T}$ and Nomura F: Titration of serum p53 antibodies in 1,085 patients with various types of malignant tumors: a multiinstitutional analysis by the Japan p53 antibody research group. Cancer 97: 682-689, 2003.

7. Sahin U, Tureci O, Schmitt H, Cochlovius B, Johannes T, Schmits R, Stenner F, Luo G, Schobert I and Pfreundschuh M: Human neoplasms elicit multiple specific immune responses in the autologous host. Proc Natl Acad Sci USA 92: 11810-11813, 1995.

8. Chen YT, Scanlan MJ, Sahin U, Tureci O, Gure AO, Tsang S, Williamson B, Stockert E, Pfreundschuh M and Old LJ: A testicular antigen aberrantly expressed in human cancers detected by autologous antibody screening. Proc Natl Acad Sci USA 94: 1914-1918, 1997.

9. Nakashima K, Shimada H, Ochiai T, Kuboshima M, Kuroiwa N, Okazaumi S, Matsubara H, Nomura F, Takiguchi $M$ and Hiwasa T: Serological identification of TROP2 by recombinant cDNA expression cloning using sera of patients with esophageal squamous cell carcinoma. Int J Cancer 112: 1029-1035, 2004.

10. Shimada H, Nakashima K, Ochiai T, Nabeya Y, Takiguchi M, Nomura F and Hiwasa T: Serological identification of tumor antigens of esophageal squamous cell carcinoma. Int J Oncol 26: 77-86, 2005.

11. Kuboshima M, Shimada H, Liu TL, Nakashima K, Nomura F, Takiguchi M, Hiwasa T and Ochiai T: Identification of a novel SEREX antigen, SLC2A1/GLUT1, in esophageal squamous cell carcinoma. Int J Oncol 28: 463-468, 2006.

12. Kuboshima M, Shimada H, Liu TL, Nomura F, Takiguchi M, Hiwasa $T$ and Ochiai T: Presence of serum tripartite motifcontaining 21 antibodies in patients with esophageal squamous cell carcinoma. Cancer Sci 97: 380-386, 2006.

13. Hiwasa T, Shimada H, Ochiai T and Takiguchi M: Serological identification of antigens by recombinant cDNA expression cloning (SEREX) using antibodies from patients with esophageal squamous cell carcinoma. In: Moleculomics and Thereafter. Hiwasa T (ed). Research Signpost, Kerala, pp99-117, 2006.

14. Van der Bruggen P, Zhang Y, Chaux P, Stroobant V, Panichelli C, Schultz ES, Chapiro J, van den Eynde BJ, Brasseur F and Boon T: Tumor-specific shared antigenic peptides recognized by human T cells. Immunol Rev 188: 51-64, 2002.

15. Rosenberg SA: Progress in human tumour immunology and immunotherapy. Nature 411: 380-384, 2001.

16. Preuss KD, Zwick C, Bormann C, Neumann F and Pfreundschuh M: Analysis of the B-cell repertoire against antigens expressed by human neoplasms. Immunol Rev 188: 43-50, 2002.

17. Shimada H, Shimizu T, Ochiai T, Liu TL, Sashiyama H, Nakamura A, Matsubara H, Gunji Y, Kobayashi S, Tagawa M, Sakiyama S and Hiwasa T: Preclinical study of adenovirus p53 gene therapy for esopahgeal cancer. Surg Today 31: 597-604, 2001.

18. Nishihira T, Hashimoto Y, Katayama M, Mori S and Kuroki T: Molecular and cellular features of esophageal cancer cells. J Cancer Res Clin Oncol 119: 441-449, 1993.

19. Oka M, Hirose K, Iizuka N, Aoyagi K, Yamamoto K, Abe T, Hazama S and Suzuki T: Cytokine mRNA expression patterns in human esophageal cancer cell lines. J Interferon Cytokine Res 15: 1005-1009, 1995.
20. Sashiyama H, Shino Y, Kawamata Y, Tomita Y, Ogawa N, Shimada H, Kobayashi S, Asano T, Ochiai T and Shirasawa H: Immortalization of human esophageal keratinocytes by E6 and E7 of human papillomavirus type 16. Int J Oncol 19: 97-103, 2001.

21. Sobin LH and Wittekind CH (eds): UICC TNM Classification of malignant tumors. 6th edition. John Wiley \& Sons, Inc., New York, 2002.

22. Nabeya Y, Ochiai T, Matsubara H, Okazumi S, Shiratori T, Shuto K, Aoki T, Miyazaki S, Gunji Y, Uno T, Ito H and Shimada H: Neoadjuvant chemoradiotherapy followed by esophagectomy for initially resectable squamous cell carcinoma of the esophagus with multiple lymph node metastasis. Dis Esophagus 18: 388-397, 2005.

23. Chomczynski P and Sacchi N: Single-step method of RNA isolation by acid guanidinium thiocyanate-phenol-chloroform extraction. Anal Biochem 162: 156-159, 1987.

24. Hsu SM, Raine L and Fanger AH: A comparative study of the peroxidase method and an avidine-biotin complex method for studying polypeptide hormones with radioimmunoassay antibody. Am J Clin Pathol 75: 734-738, 1981.

25. Verde I, Pahlke G, Salanova M, Zhang G, Wang S, Coletti D, Onuffer J, Jin SLC and Conti M: Myomegalin is a novel protein of the Golgi/centrosome that interacts with a cyclic nucleotide phosphodiesterase. J Biol Chem 276: 11189-11198, 2001.

26. Wilkinson K, Velloso ER, Lopes LF, Lee C, Aster JC, Shipp MA and Aguiar RC: Cloning of the $\mathrm{t}(1 ; 5)(\mathrm{q} 23 ; \mathrm{q} 33)$ in a myeloproliferative disorder associated with eosinophilia: involvement of PDGFRB and response to imatinib. Blood 102: 4187-4190, 2003.

27. Soejima H, Kawamoto S, Akai J, Miyoshi O, Arai Y, Morohka T, Matsuo S, Niikawa N, Kimura A, Okubo K and Mukai T: Isolation of novel heart-specific genes using the BodyMap database. Genomics 74: 115-120, 2001.

28. Nakao M, Shichijo S, Imaizumi T, Inoue Y, Matsunaga K, Yamada A, Kikuchi M, Tsuda N, Ohta K, Takamori S, Yamana H, Fujita $\mathrm{H}$ and Itoh $\mathrm{K}$ : Identification of a gene coding for a new squamous cell carcinoma antigen recognized by the CTL. J Immunol 164: 2565-2574, 2000.

29. Diesinger I, Bauer C, Brass N, Schaefers HJ and Comtesse N: Toward a more complete recognition of immunoreactive antigens in squamous cell lung carcinoma. Int J Cancer 102: 372-378, 2002.

30. Jäger E, Chen YT, Drijfhout JW, Karbach J, Ringhoffer M, Jäger D, Arand M, Wada H, Noguchi Y, Stockert E, Old L and Knuth A: Simultaneous humoral and cellular immune response against cancer-testis antigen NY-ESO-1: definition of human histocompatibility leukocyte antigen (HLA)-A2-binding peptide epitopes. J Exp Med 187: 265-270, 1998.

31. Toh U, Sudo T, Kido K, Matono S, Sasahara H, Mine T, Tanaka T, Sueyoshi S, Fujita H, Shirouzu K and Yamana H: Locoregional adoptive immunotherapy resulted in regression in distant metastases of a recurrent esophageal cancer. Int J Clin Oncol 7: 372-375, 2002. 